\title{
Refinement of parameters gravitational anomaly relative to the focal zone of the 2011 earthquake (Japan) based on satellite data
}

\author{
Tatyana Rubleva ${ }^{1 *}$, Konstantin Simonov ${ }^{2}$, Valentin Kashkin ${ }^{1}$, Anna Malkanova ${ }^{1,3}$, and \\ Roman Odintsov ${ }^{1}$ \\ ${ }^{1}$ Siberian Federal University, 79 Svobodny pr., 660041, Krasnoyarsk, Russia \\ ${ }^{2}$ Institute of Computational Modeling SB RAS, 50/44 Akademgorodok, 660036, Krasnoyarsk, Russia \\ ${ }^{3}$ Federal Research Centre "KSC SB RAS", 50 Akademgorodok, 660036, Krasnoyarsk, Russia
}

\begin{abstract}
The aim of this work is to study gravitational anomalies that have arisen in the region of the sources of strong underwater earthquakes with a magnitude of $\mathrm{M}_{\mathrm{w}}>8$. For this purpose, data obtained by the GRACE space system were used. Variations of the $E W H$ program with a period of 30 days were investigated relative to the focal area of the 2011 Japanese earthquake for the period 2010-2012. It was found that during the preparation of an earthquake, the $E W H$ values significantly increase in this area for three months, with aftershock activity, the $E W H$ values decrease within a month. Maps of variations of the $E W H$ parameter in the conditions of a disturbed geomedia and in background seismic conditions are constructed. The indices of the anomaly $\delta E W H$ were calculated, which made it possible to analyze in more detail the local gravitational field for the investigated focal zone.
\end{abstract}

\section{Introduction}

In recent years, satellite data has been widely used to analyze geodynamic activity and gravitational changes in areas of increased seismicity. Space systems GRACE (Gravity Recovery and Climate Experiment, NASA (U.S.A.) - GFZ (Germany)) and GRACE-FO (GRACE-Follow-On, of similar design, NASA/GFZ) [1,2] are successfully used to study the spatio-temporal variations of the Earth's gravitational field. Gravitation satellites of these missions have become an integral part of Earth monitoring.

The technical details of the two space missions can be found in [3]. Global Positioning System (GPS) is used to establish the precise position of GRACE and GRACE-FO satellites. The measurement error on the space system GRACE does not exceed 10 microns, and on GRACE-FO - no more than 1 microns [1].

Note that gravitational fields and their anomalies are studied using the Satellite-toSatellite Tracking (SST) technique [4]. This technique has been employed in both space systems GRACE (2002-2017) and GRACE-FO (since 2018).

*Corresponding author: tvrubleva@mail.ru 
The SST technique consists in the following: the GRACE 1 and GRACE 2 low-orbit satellites are regarded as gravimetric proof mass. Its movement is tracked to obtain the necessary data on changes in the structure of the global (or local) gravitational field over time. Gravitational anomalies observed during seismic events can also be studied using this technique.

The purpose of the present study was to refine parameters of gravitational anomalies that occurred at the epicenters of strong earthquakes of magnitudes $M_{w}>8$ that happened in the Pacific Ocean subduction zone, using GRACE data. As a case study, we considered the area of the hypocenter of the catastrophic Japan earthquake of $\mathrm{M}_{\mathrm{w}}=9.1$, which occurred at 05:46:23 UTC on March 11, 2011.

\section{Integrative processing of geophysical data}

The USGS catalog [5] was used to study geodynamic parameters of seismically active regions of strong underwater earthquakes of magnitude $\mathrm{M}_{\mathrm{w}}>8$ : the 11.03.2011 catastrophic Japan earthquake $\left(\mathrm{M}_{\mathrm{w}}=9.1\right)$, the 24.05.2013 Okhotsk earthquake $\left(\mathrm{M}_{\mathrm{w}}=8.3\right)$, and three strongest Chili earthquakes: of 27.02.2010 $\left(\mathrm{M}_{\mathrm{w}}=8.8\right), 01.04 .2014\left(\mathrm{M}_{\mathrm{w}}=8.2\right)$, and 16.09.2015 $\left(\mathrm{M}_{\mathrm{w}}=8.3\right)$.

Note that the data of monitoring gravitational anomalies observed during the strongest seismic events in these regions were analyzed using the GRACE measurements [6-8]. Analysis of the spatial structure of the anomalies confirmed the theoretical assumptions about the redistribution of the internal mass of solid Earth during geodynamic activity.

In the present study, based on integrative processing of monitoring data, maps of the epicenter of the 2011 Japan earthquake were analyzed to examine the variations in Equivalent Water Height $(E W H)$. This parameter characterizes the equivalent height above the geoid contour [9]. The data on the $E W H$ variations above the seismically active region of the catastrophic Japan earthquake with $\mathrm{M}_{\mathrm{w}}=9.1$ were found on website [1].

Software Surfer [10] was used in the stage of integrative processing of the GRACE satellite data. There were missing values in the data obtained. An accepted optimal method of interpolation is Kriging [11]. This method of geostatistics is based on statistical models and autocorrelation analysis. Kriging is fast and enables an accurate fit between the parameter values interpolated into nodes and its initial values. Finally, surfaces of spatial variables are created and their reliability is estimated.

\section{Mapping of the EWH distribution relative to the earthquake hypocenter}

The USGS the size of the focal zone of the study earthquake determined using the MMI scale was $300 \times 100 \mathrm{~km}$ [5]. For mapping and further research, we determined the boundaries of the seismically active region of the 2011 Japan earthquake: $\Delta \varphi=30^{\circ}$ $\left(25^{\circ}-55^{\circ} \mathrm{N}\right), \Delta \lambda=50^{\circ}\left(120^{\circ}-170^{\circ} \mathrm{E}\right)$.

The developed procedure was used to visualize the obtained values of the $E W H$ variations for the study region. Figure 1 shows the digital map of the $E W H$ spatial distribution relative to the region of the perturbed geo-environment of that Pacific Ocean subduction zone, including the coast of Japan. The "perturbed geo-environment" means the seismically active zone with the geodynamic conditions changed due to the seismic processes.

The "cross" in Figure 1 denotes the hypocenter of equation $\mathrm{M}_{\mathrm{w}}=9.1$ with coordinates $\varphi=38.297^{\circ} \mathrm{N}$ and $\lambda=142.373^{\circ} \mathrm{E}$. The hypocenter is $32 \mathrm{~km}$ deep. To the right of the map there is an the $E W H$ scale $(\mathrm{cm})$. 
The anomalous region for the earthquake of $\mathrm{M}_{\mathrm{w}}=9.1$ is oriented longitudinally (SouthNorth) (Fig. 1). The epicenter of the earthquake is between the positive and negative anomalies, which, to a certain extent, coincides with the segment of the boundary between the Okhotsk and Pacific plates.

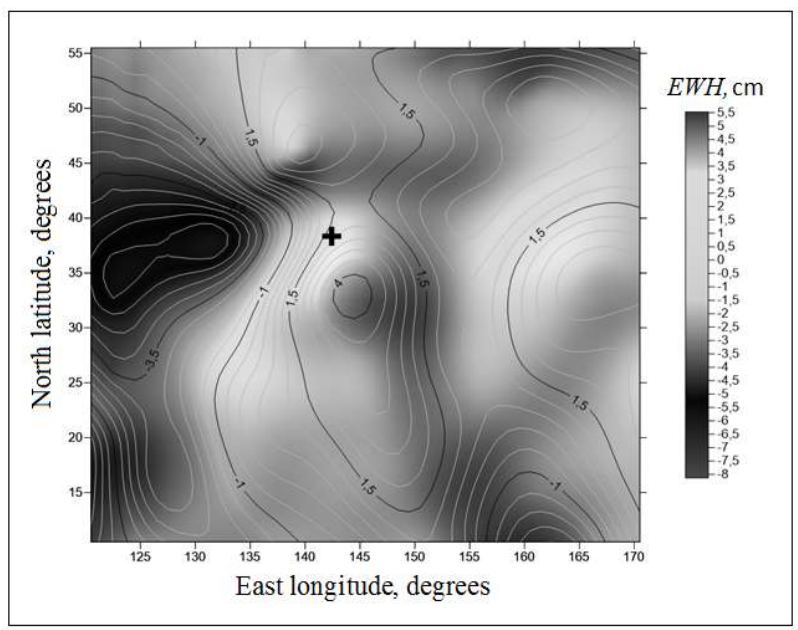

Fig. 1. Spatial distribution of the $E W H$ relative to the focal area of the Japanese earthquake

Analysis of the $E W H$ spatial distribution suggests that the hypocenter is in the zone of contour line closeness in the northern part of the positive anomalous region. In March 2011, the $E W H$ value relative to the epicenter was $1.75 \mathrm{~cm}$. The authors of [6] supposed that formation of the positive anomaly was related to the rising of the bottom of the ocean during the geodynamic activity in the region.

\section{Refining the anomaly in the zone of the Japan earthquake based on modified RST}

The USGS anomalous changes of gravitational field, to a certain extent, can be associated with geodynamic preparation processes of strong earthquakes. To refine the geometry of gravitational anomalies observed in seismically active regions of strong earthquakes by using the data on the $E W H$ variation, we employed RST (Robust Satellite Technique) [12].

Note that this technique was developed for monitoring various natural disasters including earthquakes [13]. In RST, anomaly in the spatiotemporal region is defined as deviation from the unperturbed state of geo-environment. To achieve a more accurate identification, anomaly index is calculated [14].

In the present study, employing the developed procedure, we propose using anomaly index to refine the region characterizing the gravitational anomaly observed in the perturbed geo-environment during seismic activity in the zone of the 2011 Japan earthquake. Taking into account [12], anomaly index was derived from the following formula: 


$$
\delta E W H(\varphi, \lambda, t)=\frac{\Delta E W H(\varphi, \lambda, t)}{\sigma_{\Delta E W H(\varphi, \lambda, t)}}
$$

where $\varphi, \lambda$ are geographic coordinates of the image pixel, $t$ is observation date, are values of $\Delta E W H(\varphi, \lambda, t)=E W H_{2}(\varphi, \lambda, t)-E W H_{1}(\varphi, \lambda, t)$.

Here $E W H_{2}(\varphi, \lambda, t)$ are the values of the $E W H$ during seismic activity in the perturbed geo-environment, $E W H_{1}(\varphi, \lambda, t)$ are the values of the $E W H$ measured under unperturbed geodynamic conditions, $\sigma_{\triangle E W H(\varphi, \lambda, t)}$ is the standard deviation.

Background conditions (low seismic activity) were revealed by analyzing geo-data using the USGS catalog [5]. In the study region, relatively quiet seismic conditions were observed in January 2008.

Digital maps of anomalies based on the index $\delta E W H(\varphi, \lambda, t)$ were constructed for the epicenter zone of the 2011 Japan earthquake using the developed procedure. Figure 2 shows an example of the anomaly observed relative to the epicenter zone of the Japan earthquake in March 2011. The "cross" denotes the hypocenter of that earthquake with $\mathrm{M}_{\mathrm{w}}=9.1$.

The anomaly with positive values of $\delta E W H(\varphi, \lambda, t)$ relative to the epicenter zone of the Japan earthquake was observed for four months: three months during the earthquake preparation and one month during the aftershock activity. Thus, we revealed spatiotemporal parameters of the $E W H$ variation during the geodynamic activity related to the 2011 Japan earthquake.

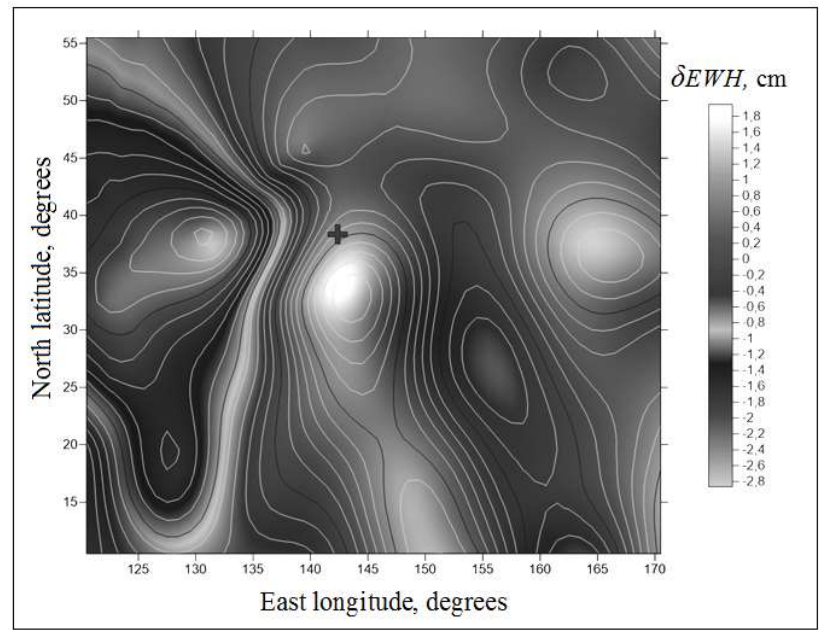

Fig. 2. The area identified by the anomaly index in the area of the 2011 Japanese earthquake

\section{Conclusion}

The present study investigated parameters of the focal zone of the Japan earthquake of $\mathrm{M}_{\mathrm{w}}=9.1$ using the $2010-2012$ data of the USGS catalog. An archive of the data based on measurements of the GRACE space system was compiled. A computational procedure was developed that enabled refining the sizes of gravitational anomalies caused by processes of 
the strongest underwater earthquakes of $\mathrm{M}_{\mathrm{w}}>8$. Satellite data were used to construct spatial models of the $E W H$ variations relative to the focal area of the 2011 Japan earthquake for the perturbed and background conditions of the geo-environment.

We studied thirty-day the $E W H$ variations relative to the focal area of the Japan earthquake for the study period. During the earthquake preparation time, for three months, the $E W H$ values were found to increase considerably in that zone, decreasing during the aftershock activity. Variations in this parameter relative to the focal area can be caused by various geophysical factors.

The modified RST was used to calculate anomaly indices $\delta E W H(\varphi, \lambda, t)$ and construct refined digital maps of gravitational anomalies relative to the study focal area. The anomaly with positive values of the index was revealed, which characterized the rising of the bottom of the ocean during geodynamic activity in the region.

We think that formation of the anomalous zone was associated with the geodynamic processes in the region. Thus, results obtained in this study suggest that measurements of the GRACE and GRACE-FO gravimetric systems can be used to analyze focal area of the strongest earthquakes.

In our future study, a representative group of the strongest earthquakes in the Pacific region will be used to analyze in detail the $E W H$ variations and relationships of this parameter to the global geodynamic and geophysical processes.

\section{References}

1. GRACE. URL: https://grace.jpl.nasa.gov/ (Accessed: 14.11.2020)

2. GRACE-FO. URL: https:/gracefo.jpl.nasa.gov/news/143/grace-fo-first-science-datanow-available/ (Accessed: 16.11.2020)

3. GFZ/ISDC. URL:https://isdc.gfz-potsdam.de/ (Accessed: 18.11.2020)

4. M.Naeimi, J.Flury, Global Gravity Field Modeling from Satellite-to-Satellite Tracking Data, Springer (2017)

5. USGS. URL: https://www.usgs.gov(Accessed: 7.12.2020)

6. V. Mikhailov, E. Timoshkina, S. Khairetdinov, P. Dmitriev, I. Kartashov, and V.Smirnov, IZV Phys. Solid Earth, 55, 5 (2019)

7. K. Simonov; V. Kashkin,T. Rubleva,K. Krasnoshekov, E3S Web of Conferences,75 (2019)

8. W. Qu, Y. Han, Z. Lu, D. An, Q. Zhang, Y. Gao, Remote Sens.,12 (2020)

9. J. Wahr, M. Molenaar, F. Bryan, J. Geophys. Res. Solid Earth,103, B 121 (1998)

10. Surfer. URL: https://www.goldensoftware.com (Accessed: 12.01.2021)

11. N. Cressie, Math. Geol., 22 (1990)

12. V. Tramutoli, V. Cuomo, C. Filizzola, N. Pergola, C. Pietrapertosa, Remote Sens., 96, 3-4 (2005)

13. A. Eleftheriou, C. Filizzola, N. Genzano, T. Lacava, M. Lisi, R. Paciello, and et al., Pure Appl. Geophys., 173 (2016)

14. F. Marchese, F. Sannazzaro, A. Falconieri, C. Filizzola, N. Pergola, V.Tramutoli, Remote Sens., 9 (2017) 